\title{
AMIDA 6 : Antiochos IV, le "Hanigalbat" et la Sophène
}

\section{Antoine Pérez}

\section{(2) OpenEdition}

1 Journals

Édition électronique

URL : http://journals.openedition.org/anatoliaantiqua/374

DOI : 10.4000/anatoliaantiqua.374

\section{Éditeur}

IFEA

Édition imprimée

Date de publication : 1 mai 2016

Pagination : 91-102

ISBN : 9782362450648

ISSN : 1018-1946

\section{Référence électronique}

Antoine Pérez, " AMIDA 6 : Antiochos IV, le "Hanigalbat" et la Sophène», Anatolia Antiqua [En ligne], XXIV | 2016, mis en ligne le 11 décembre 2018, consulté le 16 février 2021. URL : http:// journals.openedition.org/anatoliaantiqua/374; DOI : https://doi.org/10.4000/anatoliaantiqua.374

Anatolia Antiqua 


\section{ANATOLIA ANTIQUA ESKI ANADOLU}

\section{XXIV}

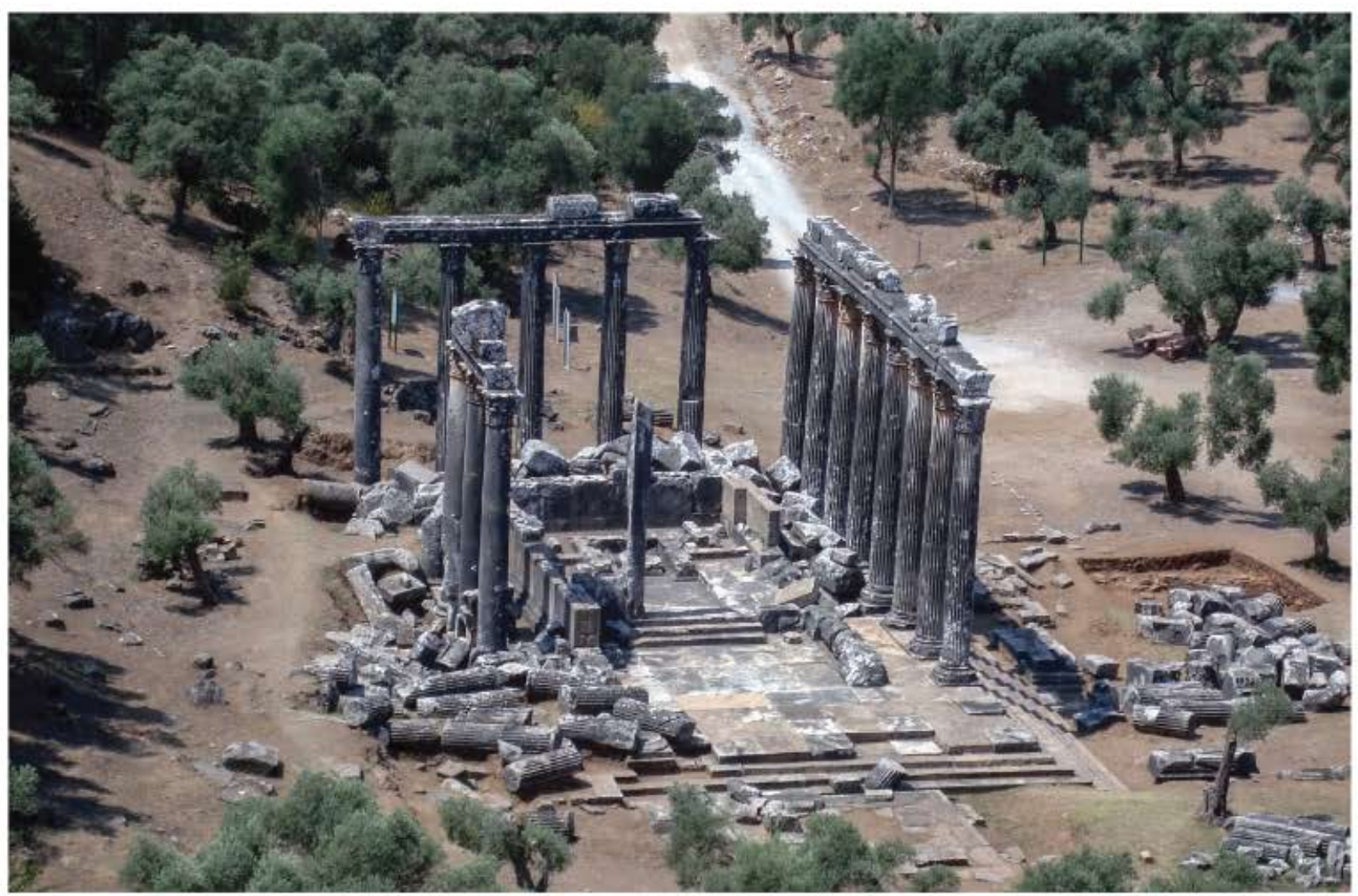

INSTITUT FRANÇAIS D'ETUDES ANATOLIENNES GEORGES-DUMEZIL

CNRS USR 3131

DE BOCCARD 


\section{TABLE DES MATIERES}

Ergul KODAS,

Le surmodelage du crâne au Néolithique au Proche-Orient : techniques de surmodelage et

expérimentations

Isabella CANEVA et Eric JEAN,

Mersin-Yumuktepe : une mise au point sur les derniers travaux

Turan EFE et Bérengère PERELLO,

Second Millenium site distribution and pottery of Inland Northwestern Anatolia

Antoine PEREZ,

Amida 6 : Antiochos IV, le "Hanigalbat" et la Sophène

Ergün LAFLI et Hadrien BRU,

Inscriptions et monuments funéraires gréco-romains d'Anatolie occidentale

Oğuz TEKİN et Aliye EROL-ÖZDİZBAY,

Coins from Allianoi excavations: Campaign of 2001

Nuran ŞAHIN,

Etude iconographique des monnaies autonomes frappées par Colophon-sur-Mer :

nouveaux acquis

Vera SAUER,

Konventionelle Individualität. Zur Münzprägung nordanatolischer Städte in der römischen

Kaiserzeit

Oğuz TEKIN,

Balance weights in the collection of the Anatolian Civilizations Museum in Ankara

\section{CHRONIQUES DES TRAVAUX ARCHEOLOGIQUES EN TURQUIE 2015}

Çiğdem MANER,

Preliminary report on the third season of the Konya-Ereğli Survey (KEYAR) 2015

Dominique BEYER, Isabelle CHALIER et Françoise KIRNER,

Rapport préliminaire sur les travaux de la mission archéologique de Zeyve Höyük-Porsuk 2015 ...

Sami PATACI et Ergün LAFLI,

Field surveys in Ardahan in 2015

Jean-Charles MORETTI avec la collaboration de Nicolas BRESCH, Isabel BONORA,

Jean-Jacques MALMARY et Olivier RISS,

Claros, le temple d'Apollon : travaux réalisés en 2015

Serdar Hakan ÖZTANER,

La basilique civile de Nysa du Méandre

Abuzer KIZIL, Koray KONUK, Patrice BRUN, Laurent CAPDETREY, Raymond DESCAT,

Pierre FROHLICH, Didier LAROCHE, Enora LE QUERE, Francis PROST, Baptiste VERGNAUD,

Eurômos : rapport préliminaire sur les travaux réalisés en 2015

O. HENRY et E. ANDERSON, Chr. BOST, Ö. ÇAKMAKLI, F. CEDERLING, A. COMMITO,

M. CORMIER-HUGUET, A. COUTELAS, A. DOLEA, D. ERGENÇ, A. FRECCERO,

A. FREJMAN, P. LEBOUTEILLER, F. LESGUER, D. LÖWENBORG, V. LUNGU,

Fr. MARCHAND-BEAULIEU, A. SITZ, P. DE STAEBLER, B. VERGNAUD, 


\section{AMIDA 6 \\ ANTIOCHOS IV, LE "HANIGALBAT" ET LA SOPHENE}

Nous avons récemment mis en perspective une mention de la Chronique dite de Zuqnin - une chronographie universelle syriaque anonyme du $8^{\mathrm{e}}$ siècle - qui nous paraît accréditer l'hypothèse, naguère initiée par Marie-Louise Chaumont, d'une fondation poliade d'Amida par le roi séleucide Antiochos IV Epiphane ${ }^{1}$. Nous voudrions développer ici quelques arguments supplémentaires propres, tout à la fois, à affermir cette proposition, et à l'insérer dans un contexte plus large : il nous paraît en effet que cet épisode mal connu de l'histoire séleucide revêt, audelà du simple cas d'Amida, une portée historique plus générale.

Rappelons les faits. En 165-164 av. J.-C., Antiochos IV se porte contre Artaxias, le satrape sécessionniste d'Arménie. Reconnu par la puissance romaine qui vient de mettre fin, à Apamée, aux ambitions occidentales des Séleucides, l'ancien stratège d'Antiochos le Grand compte bien, en effet, s'affranchir définitivement de la tutelle des rois d'Antioche. L'affaire se déroule donc dans une conjoncture historique marquée tout à la fois par le reflux de la puissance séleucide devant la montée de Rome en Orient, et par l'affirmation d'une identité dynastique arménienne promise à un long avenir.

\author{
"HANIGALBAT" : \\ ANTIOCHOS IV ET L'ARMENIE \\ DANS LES TEXTES AKKADIENS TARDIFS
}

De cette expédition arménienne, les sources classiques nous disent bien peu et c'est, de manière inattendue, à un calendrier astronomique babylonien que nous devons quelque information supplémentaire 2 . L'éphéméride consigne en effet les campagnes militaires d'Antiochos IV dans les années 165-164 av. J.-C. ${ }^{3}$ A cette occasion, l'Arménie - du moins sa partie méridionale - est assimilée à la région du Ha(b)nigalbat, dénomination assyrienne traditionnelle de l'ancien royaume du Mitanni, et qui désigna toujours, après le démembrement de ce royaume, la partie septentrionale des empires médio puis néoassyrien $^{4}-$ si l'on excepte bien sûr la parenthèse araméenne de la région, qui fit entrer Amida dans l'histoire.

\section{a. Le Hanigalbat et Amedi à l'époque protohistorique}

Il faut, pour comprendre la genèse de cette nomenclature, revenir rapidement sur l'histoire antérieure

*) Université Paul Valéry - Montpellier III, Laboratoire CRISES.

1) Assénat et Pérez 2013. La Chronique évoque la fondation d'Amida par Séleucos I ${ }^{\text {er }}$, "peu après" les villes de la Tétrapole syrienne, dans un passage inspiré de la Chronique universelle d'Eusèbe de Césarée (texte dans J.-B. Chabot I : 37, 1. 31-33). Nous avons montré que la syntaxe de la phrase impliquait sans doute l'action de l'un des successeurs de Séleucos, en l'occurrence Antiochos IV, dont la sinistre réputation interdisait qu'il fût expressément nommé par l'auteur de la Chronique, un moine originaire d'Amida. Cette fondation correspondrait à l'Epiphaneia mentionnée, "sur le Tigre", dans les Ethnica d'Etienne de Byzance.

2) Il s'agit de tablettes d'argile rédigées en cunéiforme suméro-akkadien par les prêtres de l'Esagil, le grand temple du culte poliade de Bêl-Marduk, à Babylone, depuis l'époque néo-babylonienne jusqu'au premier siècle avant notre ère (Cf. la notice introductive dans l'editio princeps de ces documents par A.-J. Sachs et H. Hunger 1988). Traduction, Del Monte 1997. Une éphéméride associe les observations astronomiques et météorologiques à quelques événements politiques et militaires se déroulant pour l'époque qui nous intéresse - dans l'empire séleucide et à ses marges. Si les rédacteurs des notices vivent à Babylone et n'ont connaissance des événements que de façon lointaine, par des informations orales, les rares renseignements qu'ils consignent sont précieux, eu égard à la pauvreté des sources classiques.

3) Le passage relatif à l'expédition d'Epiphane en Arménie puis vers le golfe Persique est consigné en AD (Astronomical Diaries) 2 : 496-497. Sur tout ceci cf. Clancier $2014: 415-417$.

4) Ibidem. Voir aussi Geller et Traina $2013: 447-454$. 


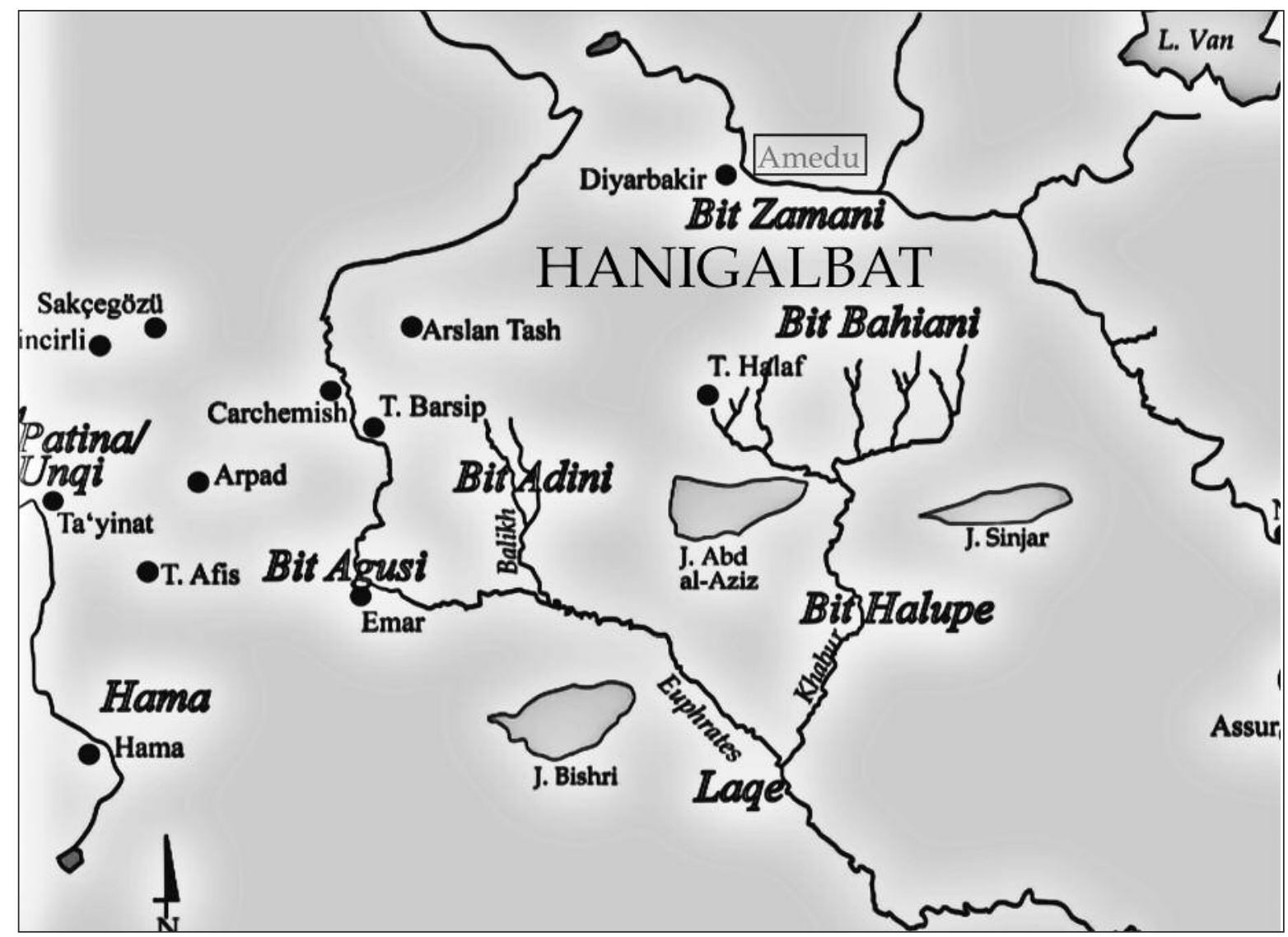

Fig. 1 : Les royaumes araméens de haute Mésopotamie au ${ }^{\mathrm{er}}$ millénaire.

de la région. On se rappellera que le royaume du Mitanni avait dominé la haute Mésopotamie entre le $16^{\mathrm{e}}$ et $1 \mathrm{l} 13^{\mathrm{e}}$ siècle. Le triangle du Khabour, au Sud-Ouest du mont Kasiiari (l'actuel Tur Abdin), en constituait le cœur - bien que sa capitale n'ait à ce jour pas été identifiée avec certitude 5 .

Après la chute du royaume, au début du $13^{\mathrm{e}}$ siècle, la haute Mésopotamie était entrée dans la sphère médio-assyrienne. Profitant de la crise interne de cet empire, au $11^{\mathrm{e}}$ siècle, les Alhamu-Araméens nomades de Syrie s'étaient peu à peu infiltrés au cœur de l'ancien Hanigalbat, vers la Djezira, vers le triangle du Khabur, le Tur Abdin puis la haute vallée du Tigre, au pied du Taurus, jusqu'aux contrées de Nairi - le futur royaume d'Urartu - aux marges extrêmes de l'ancien dominium des rois d'Assur'. Si les textes assyriens ne font état de cette infiltration continue qu'à de rares occasions, ils deviennent soudainement très diserts lorsque ces groupes, définitivement sédentarisés, constituent une série de royaumes distincts, installés dans le cadre préexistant de cités désormais abandonnées par le pouvoir d'As$\operatorname{sur}^{7}$ (Fig. 1). Centralisés sous la férule de chefs tribaux éponymes, ces royaumes sont désignés par le nom de ces sheiks : Bit (Beith) c'est-à-dire "la maison de". Ainsi des Bit-Adini, Halupe, Agusi, Bahiani ou encore du Bit-Zamani pour ce qui nous intéresse, un royaume dont la capitale, Amedi, est mentionnée pour la première fois en 866 av. J.-C.

C'est à cette date en effet, sous le règne du dynaste néo-Assyrien Assurnasirpal II, que, relatant la dix-huitième campagne du roi dans les contrées du Nord, les Annales consignent l'attaque d'Amedu, la "ville royale" du Bit-Zamani", coupable d'avoir

5) Sur le royaume du Mitanni, son extension géographique, sa chronologie, cf. Freu 2003.

6) Cf. Kupper 1956 ; Wartke 1993 ; Lipinski 2000.

7) Cf. Lipinski 2000 : passim.

8) Grayson (RIMA 2 A. 0. 101. 1) : 220. Sur le Bit-Zamani, son histoire, son extension, cf. Lipinski 2000: 135-161, et en dernier lieu Szuchman 2009. Vassal du roi d'Assur, le nouveau dirigeant du Bit-Zamani, un certain Ilanu, avait manifesté une prétention claire à l'indépendance après l'assassinat de son prédécesseur. L'expédition d'Assurnasirpal II était donc punitive. 


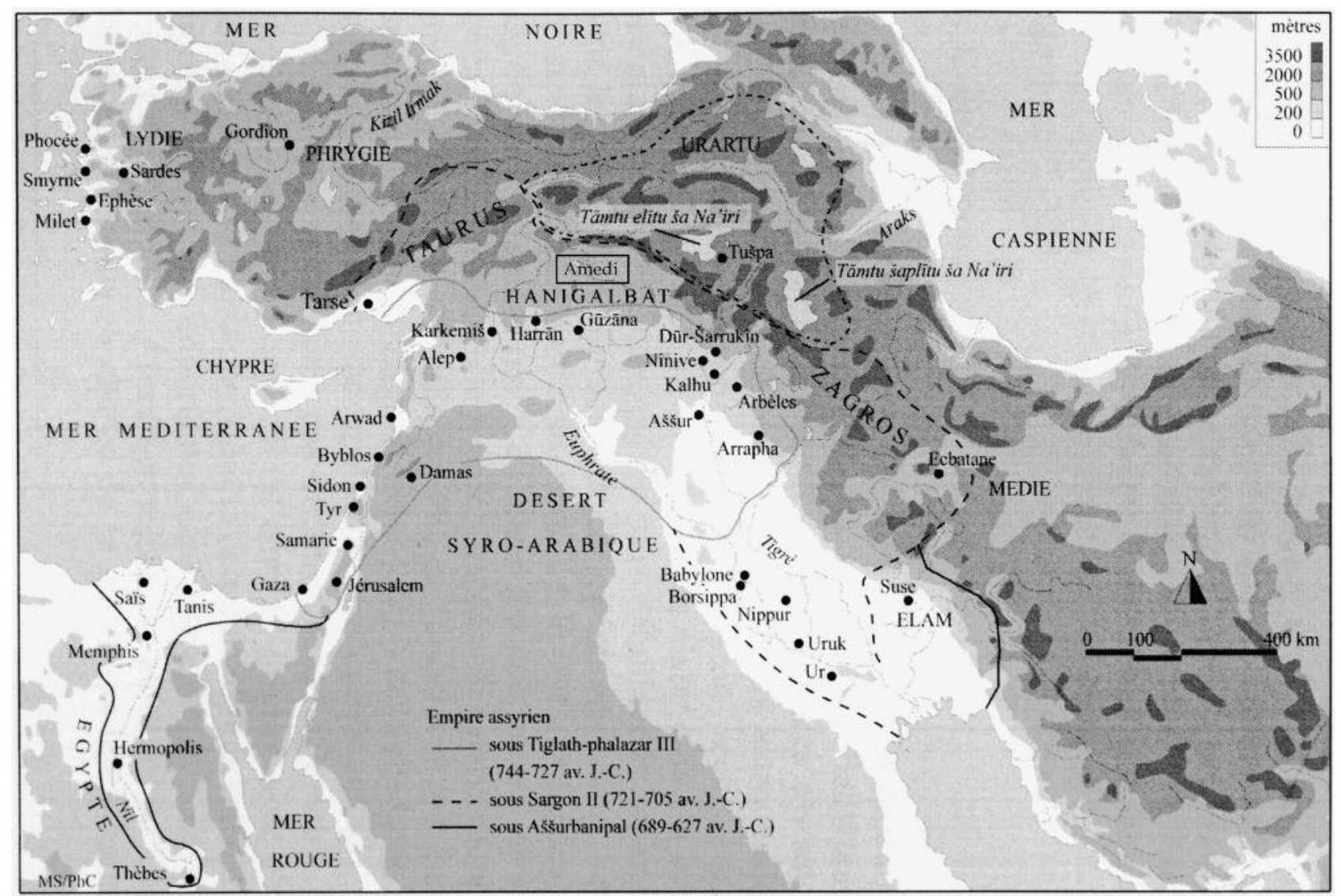

Fig. 2 : L'empire néo-assyrien et le Hanigalbat (d'après Ph. Clancier 2014, modifié).

failli à son allégeance à l'empire d'Assur. Cet événement s'inscrit dans la vaste entreprise de reconquête par les souverains mésopotamiens des provinces perdues naguère par leurs prédécesseurs ${ }^{9}$ (Fig. 2). Quelques décennies plus tard (autour de 830), le Bit-Zamani est devenu une province régulière de l'empire néo-assyrien, la plus septentrionale. Amedi en est la capitale administrative et des gouverneurs éponymes y sont attestés jusqu'à la fin du $8^{\mathrm{e}}$ siècle $^{10}$.

Or, peu avant son expédition punitive contre Ilanu d'Amedu" ${ }^{11}$ Assurnasirpal, "de retour du pays de Naïri" (au Nord) avait reçu le tribut "des rois $d u$ Hanigalbat"12, après une révolte des cités du Tur
Abdin : Amedi et le Bit-Zamani se trouvaient alors englobés dans un Hanigalbat générique qui avait perdu, dans les sources annalistiques néo-assyriennes, son sens politique et administratif au profit d'une désignation macro-régionale et culturelle ${ }^{13}$. A ce point remarquable était d'ailleurs le conservatisme terminologique des Assyriens qu'ils allèrent même jusqu'à attribuer, encore plus tard, sous le règne d'Assarhadon (680-669), le titre - purement honorifique - de "gouverneur du Hanigalbat" à un fonctionnaire, alors que cette province n'existait plus depuis le II ${ }^{\mathrm{e}}$ millénaire ${ }^{14}$.

9) Sur quoi, Garelli et Lemaire $1997: 74 \mathrm{sq}$.

10) Glassner 1993 : 164-169 ; Radner et Schadner $2001: 770-772$. Amedi est mentionnée pour la dernière fois en 705 av. J.-C. avant de plonger, comme on sait, dans un anonymat qui durera plus de mille ans.

11) Cf. supra note 8 (sécession d'Ilanu).

12) Grayson (RIMA 2) : 203 : "At that time I received tribute from Ahi-ramu, a man of Blt-Iahiri, from the (A)zallu, a man of BìtBahiāni, men of Hatti, and (from) the kings of the land Hanigalbat - silver, gold, tin, bronze casseroles, oxen, sheep, (and) horses.".

13) C'est ce que montre Fr. M. Fales (2012 : 110-111). Selon cet auteur, il s'agit bien de la région comprise entre la vallée Supérieure du Tigre, le Tur Abdin, et le triangle du Khabour. L'annalistique assyrienne utilise évidemment cet archaïsme pour souligner l'appartenance historique de la région à l'imperium des rois d'Assur. Sur l'extension territoriale du Bit-Zamani, cf. Szuchman 2009: 59 avec la fig. 1.

14) Radner $2006: 66$. 


\section{b. L'éphéméride : Hanigalbat et la Sophène}

Notre calendrier babylonien d'époque hellénistique mentionne donc pour le mois d'octobre 165 “(...) les villes du Ha(b)nigalbat, que l'on nomme Arménie (...)"15. S'il est évident que le vieux terme assyrien ne saurait ici désigner l'Arménie dans son ensemble - comme le montre le fait qu'il est utilisé de concert avec le propre terme Armini - ${ }^{16}$, il apparaît que cet archaïsme désigne bel et bien le territoire historique de l'ancien Mitanni.

Sept siècles exactement après le siège d'Amedu, à l'époque hellénistique, la zone semble donc avoir été annexée de fait au domaine arménien, qui a largement débordé au Sud du Taurus, jusqu'en haute Mésopotamie, l'ancien pays d'Hanigalbat toujours désigné comme tel dans les textes cunéiformes.

On pourrait certes objecter qu'à l'époque d'Epiphane, l'Arménie méridionale relevait stricto sensu du royaume de Sophène, dont le dirigeant Zariadris avait, à l'instar d'Artaxias, profité de la Paix d'Apamée et de l'appui romain pour se rendre indépendant ${ }^{17}$. Mais il semble bien que nos prêtres babyloniens n'aient pas suivi d'aussi près les linéaments complexes de l'histoire de cette région périphérique, ne faisant aucune distinction entre les deux branches de la Maison Orontide ${ }^{18}$. On en voudra pour preuve cette précision supplémentaire donnée par l'éphéméride quelques décennies après le règne d'Antiochos IV : en 110 av. J.-C. est consignée l'attaque de Mithridate II (de Parthie) contre l'Arménie. A cette occasion, on apprend que :

\section{"[...] ses puits (ceux du royaume d'Arménie) sont (maintenant) remplis de vase; ses toits se sont effondrés ; (tout le pays) est ruiné depuis [sa] partie supérieure [jusqu'à] sa partie inférieure, aussi loin que le Habigalbat"19.}

On constate que le Hanigalbat constitue toujours l'extension extrême, vers le Sud-Ouest, du domaine arménien, puisque le texte le distingue clairement du reste du royaume attaqué par Mithridate le Grand. Il s'agit donc bien de la partie méridionale de la Sophène, c'est-à dire la haute Mésopotamie septentrionale $^{20}$ et au premier chef la région d'Amida ${ }^{21}$. Cette année là, un successeur de Zariadris règne toujours sur le royaume, malgré les visées annexionnistes du voisin Grand-Arménien ${ }^{22}$. Il semble donc bien que la campagne d'Antiochos IV, l'admonestation

15) A D $2: 164$. Trad. Clancier : 422, en accord avec Del Monte : 80-81.

16) Clancier : 423.

17) Nommés par Antiochos III, lors de sa conquête de l'Arménie, les deux anciens stratèges avaient su, au dire de Strabon $(\mathrm{XI}, 14,5 .)^{\text {e' }}(\ldots)$ concerter leurs efforts pour s'agrandir aux dépens des nations voisines". Conjuguée à la reconnaissance officielle de ces derniers par le Sénat, l'interdiction faite au rois d'Antioche d'intervenir désormais au Nord du Taurus - une stipulation de la Paix d'Apamée, en 188 av. J.-C. - participe de la politique d'affaiblissement des Séleucides menée par Rome. - Sur la Sophène, son histoire, son extension, cf. Wheeler 2002: 87-122 (avec carte : 107); Marciak 2014

18) C. Toumanoff (1963: 292 sq.) a montré qu'il fallait ranger les deux dynasties sous une origine commune Orontide. Les rois de Sophène auraient appartenu à la "branche cadette" de cette famille (C. Toumanoff $1990: 395-396)$. En dernier lieu, M. Facella (2006 : 190-198) a montré que des dynastes orontides de Sophène ont gouverné dans le courant du $3^{\mathrm{e}}$ siècle - antérieurement à la nomination de Zariadris - un royaume séparé de la Grande Arménie, qui comportait également la Commagène avant l'accession de celle-ci à l'autonomie en 163 av. J.-C., sous la férule de Ptolémée I ${ }^{\text {er }}$ Pour un point récent de l'évolution des ces deux composantes depuis la fin de la domination achéménide et jusqu'aux règnes d'Arxtaxias et Zariadris, cf. Chaumont et Traiana, dans, G. Dédéyan (dir.) 2008 : $108-116$.

Tout cela, cependant, ne signifie pas que la Sophène était de peuplement arménien : ainsi Strabon (XI, 14, 2) désigne-il ses habitants par un ethnique, les Sophenoi, dont il situe l'origine antérieurement à celle des Arméniens, ce qui est confirmé par la mention néo-babylonienne ( $8^{\mathrm{e}} \mathrm{s}$.) du royaume septentrional de Supani (Weissbach 1927 : 1016). En dernier lieu, Marciak 2014 : 18, après un bilan serré : "...we can suggest that the origin of Sophene is not Armenian in character". Mais c'est un autre sujet.

19) A D 3 : 110. Trad. de Geller et Traina (2013 : 449) reprise par Clancier (2014: 427) que nous reproduisons ici. Contra, Del Monte (1997 : 153-154) postule l'équivalence Hanigalbat-Armini.

20) Si la frontière septentrionale de l'ancien Mitanni n'est pas connue de manière précise - on ne saurait dire si elle a outrepassé de beaucoup la chaîne du Taurus, vers le Nord -, il est de toutes façons évident que le Hanigalbat désigne exclusivement la haute Mésopotamie, en deçà du Taurus.

21) L'appartenance de la région d'Amida à la Sophène est sûre, ce qui est prouvé par Pline $(H . N ., \mathrm{V}, 66)$ et surtout par Plutarque (Lucullus, XXIV, 4-8) dans le contexte de la campagne mithridatique de l'année 69 av. J.-C. : le général romain, en route vers la capitale de Tigrane où s'est réfugié Mithridate, avance “à travers la Sophène” après avoir traversé l'Euphrate, montrant à ses soldats le Taurus "dans le lointain", sur leur gauche en progressant vers le Tigre. Lucullus et son armée sont alors sur le plateau de Diyarbakır, après avoir franchi la passe d'Arghana-Maden, et empruntent la Route Royale de Darius dans la direction de Tigranocerte, l'actuelle Silvan, à quelque $70 \mathrm{~km}$ à l'Est de Diyarbakır. A moins qu'ils n'aient coupé plus au Nord, mais toujours dans la région de Diyarbakır. Pour un examen détaillé des itinéraires possibles, cf. Eckardt 1910 : 84-88. En dernier lieu, cf. la carte dans l'atlas de la Neue Pauly (2012: 159).

22) Annexionnisme qui s'expliquerait donc par la parenté entre les deux satrapes dont on a parlé supra, Artaxias n'ayant voulu en fait que procéder à une réunification (sur quoi, Mutafian et Van Lauwe 2001 : 26). Quoi qu'il en soit, on ne sait plus rien de Zariadris après sa reconnaissance par les romains en 188. Artaxias n'eut de cesse - en vain - de tenter de mettre la main sur la Sophène après la 


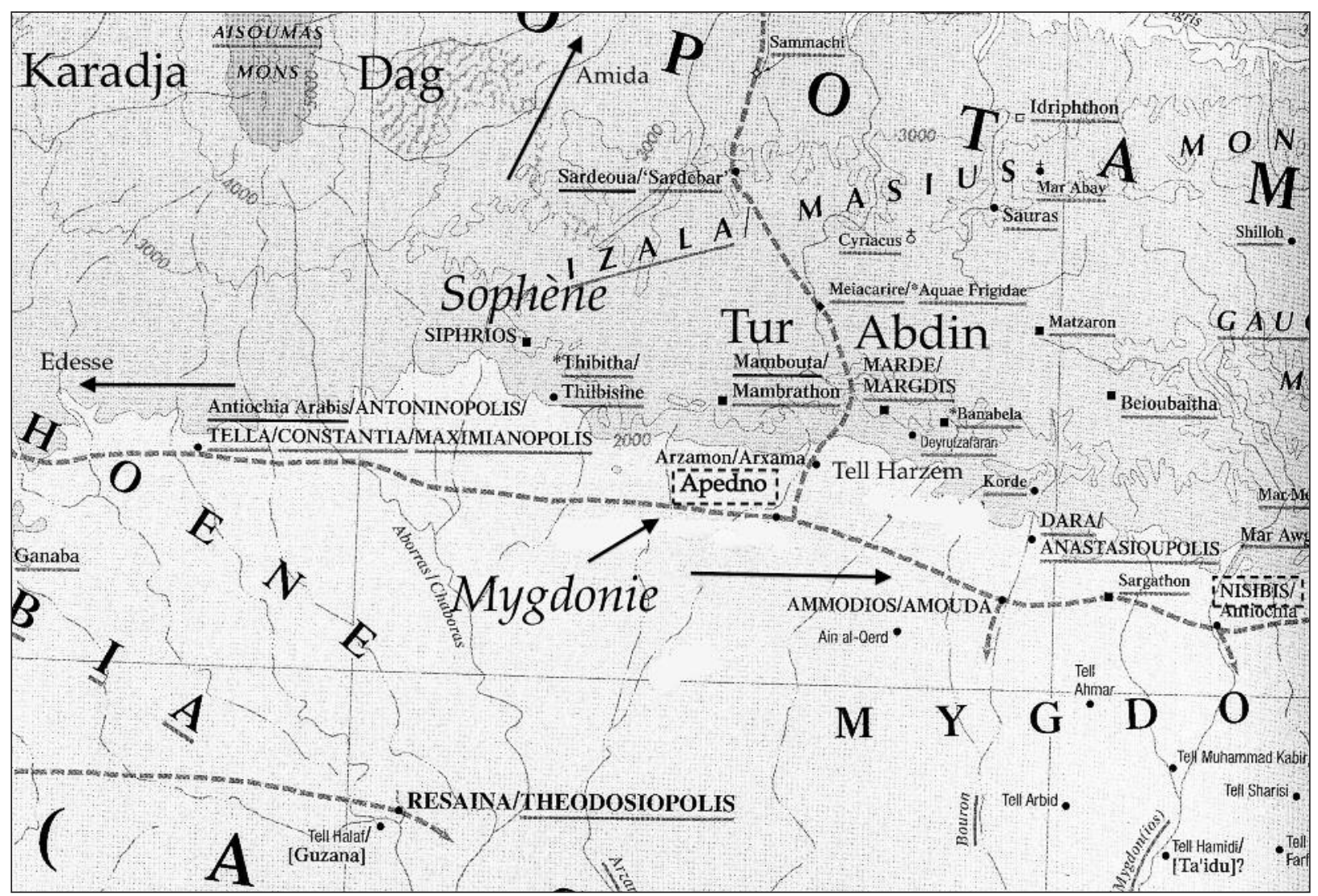

Fig. 3 : Frontière méridionale de la Sophène au $2^{\mathrm{e}}$ S. av. J.-C. et localisation d'Apedno, en Mygdonie (Fond: Atlas Barrington, pl. 89, modifié).

d'Artaxias et la fondation d'Epiphaneia n'avaient pas changé véritablement la donne en haute Mésopotamie - à ceci près que, si dans la première moitié du $2^{\mathrm{e}}$ siècle l'administration de la région par un dynaste arménien ne contrevenait pas, en théorie, à la tutelle séleucide ${ }^{23}$, à la fin du même siècle, elle signifiait clairement la perte irrémédiable de ce territoire.

Les textes cunéiformes confortent donc un constat que les sources classiques avaient déjà permis d'inférer : la présence arménienne en haute Mésopotamie est manifeste au $2^{\mathrm{e}}$ siècle avant notre ère, même s'il est bien difficile d'en fixer la limite méridionale, et en particulier de savoir - on va y revenir - si elle atteignait au triangle du Khabour et à la Jezirah, audelà même des frontières présumées de la Sophène ${ }^{24}$ (Fig. 3). On comprend en tout cas que, au rang des motivations qui poussèrent, en 165, Antiochos Epiphane à intervenir "au Hanigalbat", figurait au premier chef le souci de contenir les anciens stratèges au-delà des montagnes (le Taurus) marquant la frontière "naturelle" du royaume syrien, avant de leur confier plus pragmatiquement la garde de cette marche, en qualité de vassaux ${ }^{25}$. Et ce d'autant que, pour les raisons qu'on a dites précédemment, le roi

mort de son ancien allié (ou celle de son successeur). Elle ne fut annexée que par Tigrane II qui détrôna son dernier roi, Artanès, en 95 av. J.-C. (Strabon, XI, 14, 15). La Sophène fut alors intégrée dans le grand empire arménien de Tigrane auquel Lucullus puis Pompée devaient mettre fin dans les année 60 av. J.-C.

23) L'attitude d'Antiochos IV à l'endroit d'Artaxias est conforme à la politique séleucide de vassalisation de stratèges-dynastes dont L. Capdetrey (2007 : 130 sq.) fait une clé du contrôle séleucide dans les régions périphériques... en même temps que de sa fragilité, ici particulièrement évidente. Ce mode de contrôle indirect était d'ailleurs le seul possible pour des territoires enclavés aussi difficilement assimilables que l'Arménie, la Sophène ou la Commagène, ainsi que le précise M. Facella (op. cit. : 169).

24) Sur les marges méridionales du royaume, cf. infra.

25) Peut-être faudrait-il ici employer le singulier puisque seul Artaxias est mentionné par les sources. Ainsi, dans son tableau des satrapies du royaume, L. Capdetrey (2007 : 235) attribue-t-il une satrapie d'Arménie/Sophène à ce seul titulaire sous le règne d'Antiochos IV. On est pourtant bien obligé de prendre en compte l'existence d'un roi de Sophène dont on ne connaît pas le nom en 165-164 av. J.-C. - ce n'est plus Zariadris, ce n'est pas encore son fils Mithrobarzane - puisque, comme on l'a vu supra, la Sophène est toujours indépendante dans les sources jusqu'en 95 av. J.-C. 
d'Antioche avait sans doute fait le deuil des possessions occidentales de son empire, privilégiant désormais la consolidation de ses marges septentrionales et orientales ${ }^{26}$. Pour ce faire, il apparait comme logique que le Séleucide ait jalonné par une fondation poliade et royale sa marche vers le royaume séditieux, marquant de fait officiellement l'appartenance politique de la région à son domaine, en une zone où la frontière de l'état séleucide ne semblait jamais avoir été fixée - ou officiellement définie - de manière très précise ${ }^{27}$.

\section{APEDNO/APÂDANA....}

Comme on l'a vu dans notre précédent article ${ }^{28}$, Marie-Louise Chaumont a localisé la bataille décisive opposant Epiphane à Artaxias, s'appuyant sur un fragment de Porphyre qui donne le lieu de campement du roi syrien suite à la bataille : Apedno (ou Apadna $)^{29}$. Le site, qui a connu diverses appellations ${ }^{30}$, doit être identifié à la localité moderne de Tell Harzem ${ }^{31}$, au pied du Tur Abdin, un peu au Nord du triangle du Khabour, c'est-à-dire en plein "Hanigalbat", pour reprendre la nomenclature babylonienne. Sise au cœur de la haute Mésopotamie, au carrefour des axes reliant respectivement Edesse à Nisibe, et la plaine syrienne au decht de Diyarbakır, Apedno affectait une position remarquable, juste au contact du mont Masios ( $\mathrm{Ka}$ radja-Dag et Tur Abdin, l'ancien Kasiiari des Assyriens), et donc de la limite méridionale extrême du domaine arméno-sophénien (Fig. 3) 32 .

Comme on sait, le terme apâdana désigne, en perse, la salle d'audience du palais et, partant, la résidence d'un gouverneur représentant l'autorité royale ${ }^{33}$. Apedno avait, semble-t-il, gardé cette fonction administrative sous les Séleucides puisque Pline l'Ancien l'appelle significativement Regia Satraporum, précisant qu'elle était "jadis" très étendue et qu'on y percevait le phoros $^{34}$. L'emprise disproportionnée du site - un périmètre de 70 stades selon le naturaliste, soit plus de douze kilomètres - a conduit Louis Dillemann à postuler l'existence, à Apedno, d'un paradeisos, vaste jardin public qui agrémentait tous les centres de gouvernement provinciaux achéménides et, dans certains cas au moins, séleucides ${ }^{35}$.

Ce renseignement capital a été, nous semble-til, sous-estimé par les chercheurs ${ }^{36}$. Il ne saurait en effet être anodin que l'affrontement décisif se soit déroulé en ces parages. Antiochos IV a manifestement dressé son campement là où devait se trouver l'administration régionale séleucide de la contrée, comme

26) Assénat et Pérez 2013 : 163.

27) Sur le caractère mouvant des frontières séleucides, cf. Capdetrey 2007 : 130 sq. Flou que traduisent les différences notables entre les cartographies du royaume séleucide dans les atlas modernes.

28) Assénat et Pérez 2013 : 164.

29) Porphyre apud Jérôme, Commentaire à Daniel (11, 44-45), (Patr. Lat. XXV, c. $573=F r$. Gr. Hist., éd. Jacoby, II B 260, frag. 56 : 1227-1228) : "'...) Confestimque perget ad Artaxiam regem Armeniae, qui de orientis partibus mouebitur, et interfectis plurimis de eius exercitu ponet taberbaculum in loco Apedno, qui inter duo latissima situs est flumina, Tigrim et Euphratem". Voir Chaumont $1993: 435$.

30) Apedno donc, chez Porphyre ; Apadna dans la Notitia Dignitatum (Or., XXXV) et chez Procope (de aed. II, 4, 20) ; Aphadhana chez Zacharie le Rhéteur (cf. note suivante); Arxama chez Ptolémée (V, 18, 11); Arcamo sur la Table de Peutinger (Sect. XIII de l'éd. K. Miller); Caphrena, auparavant Regia Satraporum chez Pline l'Ancien (VI, 30, 119), sur lequel nous revenons de suite.

31) C'est Zacharie le Rhéteur (Hist. Eccl., VI, 5 : 21 dans l'éd. Brooks) qui fonde cette identification, évoquant, lors du conflit persique de 503 ap. J.-C., la présence d'une armée romaine "(...) a pago Harzam, qui ad Aphadhana pertinet A(e)rebindus". Voir encore Dillemann : $159 ; 245$.

32) Sur les frontières de la Sophène cf. la carte dans Hewsen 2000, pl. 23. Egalement, Mutafian et Van Lauwe 2001 : 27 ; en dernier lieu, Wheeler 2002.

33) Meillet et Benveniste 1931 : 156. Sur l’Apâdana de Darius et Xerxès, à Suze, celui de Persépolis, cf. Briant, op. cit. : $178-182$. Cette désignation s'est étendue par métonymie aux centres de pouvoir régionaux.

34) VI, 30, 119 : "(...) ex qua (Apameam) orientem petentes excipit oppidum Caphrena munitum, quondam stadiorum LXX amplitudine et satraparum regia appellatum, quo tributa conferebantur, nunc in arcem redactum ". On aimerait savoir exactement ce que recouvre ce quondam. Mais le passage s'inscrit dans un contexte (le chapitre trentième) relatif au réseau urbain proprement macédonien, depuis Alexandre et ses successeurs. Selon Dillemann (op. cit. : 165-170) la description Plinienne suit une une route indiscutablement séleucide. Pierre Briant (op. cit. : 207) a utilisé "non sans réserves" ce passage pour documenter la pratique achéménide de la cérémonie de remise des tributs (le phoros). Mais il s'interroge moins, semble-t'il, sur la réalité hellénistique de ce renseignement que sur sa pertinence pour l'époque antérieure.

35) Op. cit. : 170. Sur les paradis provinciaux dans l'empire de Darius, cf. Briant, op. cit. : 98-99; $245-251$; 456-459. Combinant parc d'agrément naturaliste et production fermière, ces paradis provinciaux pouvaient affecter une très vaste emprise (Liverani 2008 : 328). Si aucun texte ne nous informe sur le devenir hellénistique des paradis achéménides provinciaux (Capdetrey 2007: 145 sq.), il semble bien que les capitales satrapiques, du fait de leur fonction de résidences royales, en furent toujours pourvues, de même que des centres plus secondaires (ibidem : 375).

36) A l'exception, donc, de L. Dillemann qui n'en a cependant pas analysé toutes les implications. 
elle devait l'être précédemment sous les Perses ${ }^{37}$. Lieu de stationnement tout indiqué pour une armée en campagne, le paradeisos, avec son système d'irrigation généreux ${ }^{38}$, a dû pourvoir les troupes en eau et ressources vivrières dans cette région marquée par l'aridité. Un tel fait n'est pas inédit: Xénophon ${ }^{39}$ décrit ainsi la revue des troupes (13000 hommes) à laquelle Cyrus le Jeune avait naguère procédé dans le paradis de Kelainai, en Grande Phrygie, cependant qu'au témoignage de Quinte-Curce ${ }^{40}$, Alexandre dîna "avec toute son armée" dans un paradis de Sogdiane. C'est dire l'ampleur de ces édens, tout à la fois jardins horticoles, bois d'agrément et parcs animaliers qui, s'ils n'affectaient peut-être plus sous les rois macédoniens le faste de l'époque achéménide, devaient encore - du moins pour certains - constituer des lieux propres à satisfaire les besoins d'une halte ou d'un stationnement militaire. Les mentions de Pline et de Porphyre éclaireraient donc tout à la fois les circonstances du campement à Apedno - le stationnement dans le paradis - et ses raisons - la défense d'un chef-lieu provincial ${ }^{41}$.

Mais de quel chef-lieu, et de quelle "province" s'agit-il?

En dehors d'Apedno, au moins trois localités homonymes sont connues en Syrie-Mésopotamie ${ }^{42}$ (Fig. 4). Toutes n'étaient peut-être pas des centres administratifs : on sait qu'une demeure royale sur une route officielle achéménide pouvait porter ce nom $^{43}$. L'une de ces localités, cependant, intéresse tout particulièrement notre propos, qui se trouvait à quelques trois cents kilomètres au Sud de notre Apedno, non loin de Doura Europos : il s'agit d'A(p)p(h)adana, connue par des papyrus du MoyenEuphrate au $3^{\mathrm{e}}$ siècle ap. J.-C. ${ }^{44}$. Cette agglomération de la Coelé-Syrie romaine, sise sur la rive droite de l'Euphrate, non loin de son embranchement avec le Khabour, joua encore à l'époque impériale un rôle administratif éminent : c'était le centre d'un ressort fiscal et judiciaire - dioikièsis - où siégeait un fonctionnaire équestre de rang procuratorien ${ }^{45}$. Il n'est pas anodin qu'une agglomération d'aussi modeste importance - si on la compare aux cités voisines ait joué un tel rôle, qui finit par lui valoir une promotion au rang de cité, sous le nom de Neapolis ${ }^{46}$ : ce fait ne peut être expliqué que par la perpétuation en un même lieu d'une même fonction, fait assez fréquent dans le monde romain.

Il serait certes hasardeux de faire fond sur un rapprochement mécanique entre les sites et les époques : il reste qu'on ne peut que souligner la remarquable identité de fonction des deux Apâdana la diokièsis à Appadana ; la perception du phoros (sous la responsabilité d'un dioikètes) à Apedno -, qui semblent bien avoir conservé à travers le temps le rôle administratif que désigne leur onomastique perse.

37) Cette importante question de la pérennité des fonctions et des centres administratifs sous les Séleucides est âprement discutée, la documentation étant difficile à interpréter (cf. la mise au point de Capdetrey 2007 : 229-231), mais la communis opinio penche pour une adaptation globale au système mis en place par les Achéménides. Sur quoi, L. Capdetrey $2008: 134$ (cette continuité est avérée en Asie Mineure). On doit cependant prendre en compte l'évolution complexe et les réorganisations nombreuses tout au long de l'histoire du royaume séleucide, dont la stabilité ne fut pas le point fort. La présence d'un paradis à Apedno plaide en tout cas, pour ce qui est de notre région, dans le sens d'une telle continuité.

38) Sur la source de Tell Harzem, cf. Dillemann :170.

39) Anab. I, 2.9.

40) Hist. VIII, I.19.

41) On ne voit en effet guère ici de raison de dissocier les deux : si le paradis existait encore, c'est qu'il y avait un centre administratif, à tout le moins un lieu de pouvoir.

42) Chaumont 1984. Point plus récent sur la localisation de ces Appadana dans Gnoli 1999 : 321-358; 2000 : 31-66.

43) Chaumont 1986-1987 : 296. Mais, à l'époque, l'auteur n'avait pas connaissance des papyrus publiés par Feissel et Gascou (cf. note suivante).

44) Cf. Feissel et Gascou 1989 : 535-561. Deux localités homonymes de même orthographe mentionnées par Ptolémée "en Mésopotamie" : l'une sur l'Euphrate, au Sud de son embranchement avec le Khabour (V, 18, 6) ; l'autre, plus au Nord, sur le Khabour (V, 18,13 ). Les auteurs se prononcent pour la première (ibid. : 542), les documents publics du dossier papyrologique "Moyen-Euphrate" évoquant en effet exclusivement la juridiction du gouverneur de Coelé-Syrie à Antioche (or, l'Appadana du Khabour, à cette époque, est en Osrhoène). Selon Feissel et Gascou (ibid. : 541), la localité mentionnée dans les "archives" de Doura et celle des papyrus récemment découverts ne font qu'une. Là se trouvait stationné un corps de troupes auxiliaires détaché de la $\mathrm{XX}^{\mathrm{e}}$ cohorte des Palmyréniens, au témoignage d'un papyrus de Doura ( ${ }^{\circ}$ 60) jadis publié par M. Rostovtzeff (CRAI 1933: 309-323) et de quelques autres. Contra, T. Gnoli opte d'abord (1999 : 354-355) pour l'Appadana/Basileia sise un peu plus au Nord, sur l'Euphrate, près de Zalebiyeh ; puis (2000: 64) pour l'Appadana du Khabour. Mais cela ne change rien à notre propos.

45) Feissel et Gascou 1995 : 72 : une pétition adressée au gouverneur de Coélé-Syrie, relative à une usurpation de domaine, mentionne "(...) Claudius Ariston, vir egregius, procurateur siégeant à Appadana de qui dépend la dioikèsis (...)”. Cette dioikèsis, selon les auteurs (84) serait une sorte de conventus judiciaire que le fonctionnaire impérial aurait tenu en lieu et place du gouverneur. Il reste qu'on ne peut être que frappé de la nature de cette juridiction d'époque romaine, qui semble bien perpétuer en partie la fonction hellénistique du dioiketès, lequel prononçait justement sur les questions fiscales et cadastrales (cf. Capdetrey 2007 : 312-314).

46) En 244-255 ap. J.-C. ou un peu plus tôt, selon M. Sartre 2001. Cf. Feissel et Gascou 1995 : 542 ; Gnoli : $354-355$ (avec un désaccord sur l'identification). On n'écartera pas, pour cette promotion, l'importance du facteur militaire. 


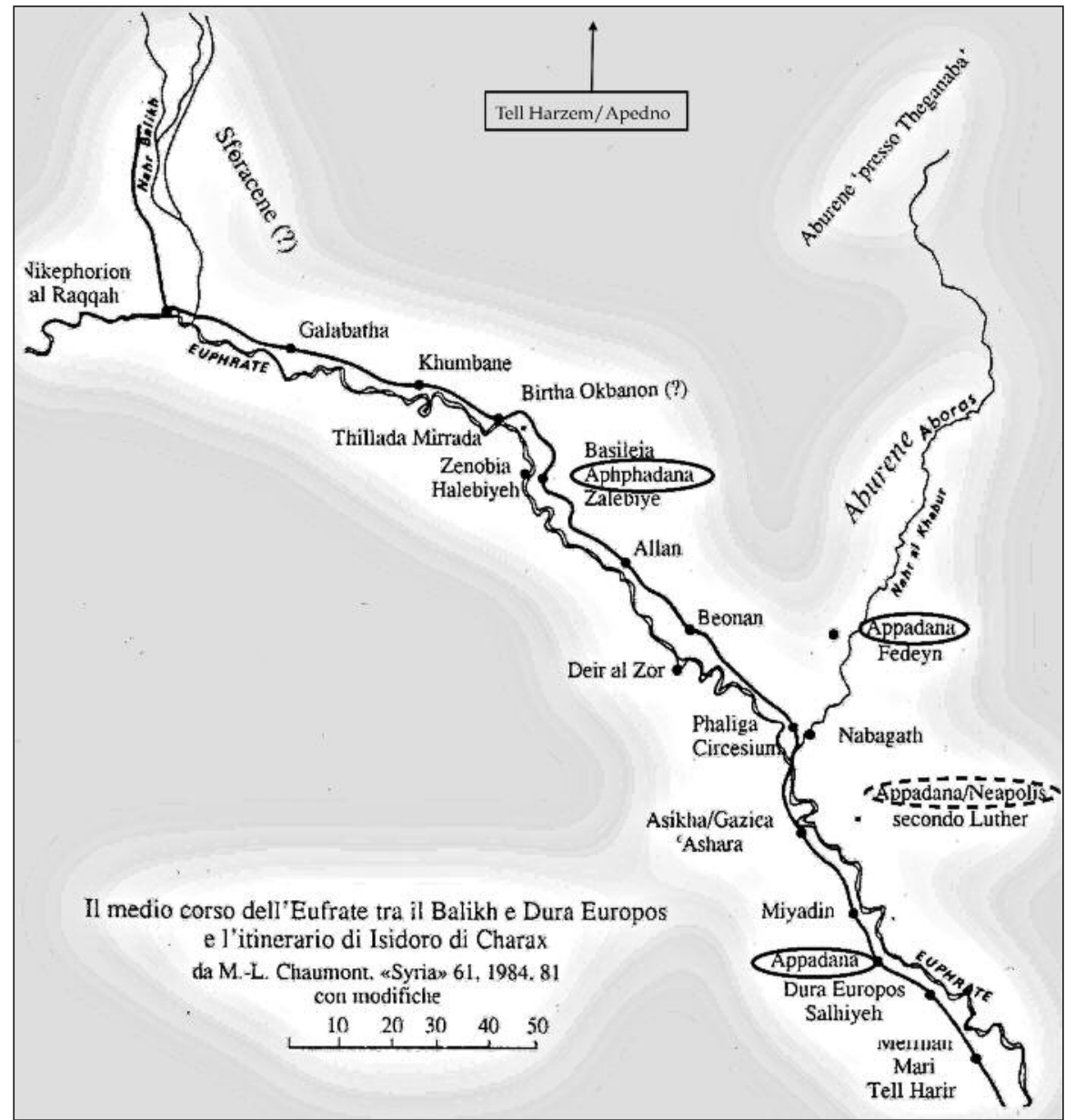

Fig. 4 : Localisation des Appadana du Moyen Euphrate

(d'après T. Gnoli 2000, modifié).

On n'insistera pas davantage sur le cas de la troisième Appadana syrienne qu'Isidore de Charax appelle, de manière significative, Basileia ${ }^{47}$, probablement à identifier avec l'agglomération sise près de Zalebiyeh, sur l'Euphrate ${ }^{48}$, l'onomastique étant, là encore, on ne peut plus suggestive.

Sous les Séleucides, les deux - ou trois - Appadana "méridionales" relevaient, jusqu' au milieu du $2^{\mathrm{e}}$ siècle, d'une éparchie dont l'identité n'est pas claire, au Nord de la Seleukis Syria : peut-être la Mésopotamie/Parapotamie ${ }^{49}$. Plus septentrional, notre
Apedno appartenait à l'éparchie de Mésopotamie/ Mygdonie ${ }^{50}$ ou à l'une de ses subdivisions districales ${ }^{51}$. Décrite par Strabon ${ }^{52}$, cette satrapie s'étendait au Sud de la ligne du Masios (Karadja-Dag et Tur Abdin), depuis l'Euphrate jusqu'au Tigre, et comprenait, outre Nisibe, les villes d'Harran (Carrhae) et Nikephorion/Tella (Fig. 3). On peut donc écarter fermement l'hypothèse qu'Apedno ait pu appartenir à la satrapie septentrionale d'Arménie/Sophène également décrite par Strabon $-^{53}$ laquelle, rappelons-le, était une entité périphérique de l'empire sé-

47) FgrHist 781 F 2.

48) C'est cette Appadana que, comme on l'a vu supra, T. Gnoli identifie d'abord (1999: 355) à l'agglomération des papyrus de l'Euphrate.

49) Capdetrey $2007: 250-251$, avec des réserves.

50) Tableau des satrapies séleucides dans Capdetrey (2007: 232-235). Sur la création précoce du district de Mygdonie “dans le prolongement des antécédents pré-séleucides", ibidem : 251. On ne discutera pas de la nomenclature précise de ces unités territoriales séleucides : satrapies, stratégies ou encore éparchies sont en effet interchangeables dans les sources (ibidem : 231).

51) C'est l'hypothèse de M-L. Chaumont 1986-1987: 296.

52) XI, 14, 2 ; XVI, 23 (pour une époque difficile à préciser, mais c'est le seul texte disponible avec Pline, cité plus haut).

53) XI, 14, 2 . 
leucide, administrée par le dynaste lui-même, non par un gouverneur dépêché d'Antioche ${ }^{54}$. La mention de Porphyre pourrait alors conserver précisément le souvenir d'une bataille pour le contrôle d'un centre provincial séleucide dont on ne saurait dire s'il était déjà, au début de l'engagement, aux mains des Arméniens, ou bien en danger d'annexion imminente. Il ne pouvait être, en effet, question de laisser des rois étrangers, de surcroît désormais soutenus par la puissance romaine, s'implanter si profondément en pays mésopotamien : si le territoire de la Sophène englobait à cette époque la Gumathène et la région d'Amida au Sud du Taurus, jusqu'au Masios, le contrôle par les Orontides de l'un des chef-lieux de la satrapie "macédonienne" de Mygdonie - et de ses ressources fiscales - ne pouvait être perçu que comme une inadmissible violation.

C'est donc probablement à cette occasion, selon nous, qu'Antiochos attribua à Nisibe le nom d'Antioche de Mygdonie ${ }^{55}$. Sise au piémont sud du Tur Abdin (Masios) dont, au dire de Strabon, "...le versant méridional domine la Mygdonie - c'est-àdire le canton de la Mésopotamie où est Nisibe" 56 , la cité se trouvait en des confins dangereux. Peutêtre même avait-elle déjà été annexée, ce que nous ne savons pas. Quoi qu'il en soit, le roi aurait réaffirmé, par cette paronymie macédonienne, que Nisibe - et par là toute la Mygdonie - demeurait partie intégrante du domaine séleucide. Dans le même temps il reprenait la main, au Nord du Masios, sur toute la Sophène méridionale sub-taurique, en l'occurence le plateau d'Amida/Diyarbakır, assortissant cette prise de possession d'une fondation nouvelle : Epiphaneia $^{57}$.

Le fait que la bataille se soit déroulée à cette latitude montre bien à quel point les Arméno-Sophéniens s'étaient profondément avancés vers le Sud : les implications de cette mention de Porphyre - passée un peu inaperçue - sont alors lourdes de sens, qui dépassent de loin le seul cadre alto-mésopotamien. C'est que la question semblait en effet déjà moins, en 165, de ramener à l'obéissance Artaxias et son allié sophénien en Arménie - on vient de voir que la cause était entendue depuis plus de vingt ans - que de colmater, aux portes mêmes de la Syrie, une brèche béante menaçant le cœur du domaine séleucide : on n'aura garde d'oublier que, depuis le verdict de Magnésie, la figure royale arménienne tendait à se confondre avec la menace romaine ${ }^{58}$. Dans ces conditions, 1"eanabase" d'Antiochos IV Epiphane doit être considérée moins comme une marche triomphale ${ }^{59}$ que comme une expédition défensive dictée par l'urgence : le début, en fait, de la longue agonie de l'"homme malade" de l'Asie hellénistique ${ }^{60}$.

\section{AMIDA... OU $A N G L$-EGIL ?}

De ce contexte géographique s'ensuit en tout cas que, Apedno ne se trouvant pas sur le Tigre, seules deux villes peuvent valablement candidater au titre d'Epifaneia kata Tigre: Amida et Angl (nom arménien d'Egil), l'antique ville de Sophène, à quelques quarante kilomètres au Nord de Diyarbakır, sur le cours supérieur du fleuve ${ }^{61}$. Comme nous l'avons vu dans notre précédent article, la mention du moine de Zuqnin nous semble aujourd'hui faire pencher le plateau de la balance plutôt en faveur $\mathrm{d}^{\prime}$ Amida $^{62}$.

Dans ce sens va également la disposition topographique très particulière d'Angl/Egil. Le site est connu comme culturellement arménien : c'était un important sanctuaire païen dédié à Tork' d'Angl, version arménienne de Nergal, la vieille divinité mésopotamienne des enfers ${ }^{63}$. La forteresse d'Angl abritait en outre la nécropole royale des Orontides,

54) Cf. Capdetrey 2007 : 130-132. M.-L. Chaumont (1984 : 296) écarte également la possibilité que Tell Harzem et Nisibe aient été englobés dans la satrapie d'Arménie (c'est-à-dire de Sophène). On admettra donc comme probable la frontière telle que définie par Wheeler 2001, fig. $23 ; 2002: 89$ sq.

55) Assénat et Pérez 2013 : 163 note 37. Selon G. Le Rider (1994 : 23-24), le terminus post quem des frappes nisibéennes est l'année 169/168. On en restera, malgré T. Bousdroukis (2003: 12-13 note 10) à l'opinion la mieux partagée, que résume L. Capdetrey (2007 : 74 note 139) : si la fondation de Nisibe est l'œuvre de Séleucos - après, comme on l'a dit plus haut, une éventuelle action d'Antigone le Borgne - la métonomasie de Nisibis en Antioche de Mygdonie est probablement due à Antiochos IV, malgré une mention anachronique de Polybe (V, 51,1). Le récent ouvrage de G.M. Cohen (2013 : 62-67) n'apporte rien de nouveau.

56) XI, 14, 2.

57) Epifaneia ... kata Tigre, selon Etienne de Byzance (Ethnica, s.v. Epifaneia).

58) Strabon, XI, 14, 15: le Sénat a reconnu les deux anciens stratèges. Sur quoi, Frankfort $1963: 182$ sq.

59) L'expression est de P.F. Mittag $2006: 208$ note 108.

60) Pour reprendre le mot de L. Capdetrey $2007: 11$.

61) Sur le nom du site dans les sources, cf. Assénat et Pérez $2013: 164$ note 49

62) Ibidem : passim.

63) C'est ainsi que l'appelle la version arménienne de la Bible (Rois, XVII, 30). D'où le nom arménien de la région d'Egil dans l'antiquité, l'Angel Tun, c'est-à dire 'la maison du dieu Angl'. Sur l'équivalence avec Nergal, cf. Petrosyan $2002: 13 s q$ 
unificateurs historiques du premier royaume d'Arménie, ainsi qu'une partie de leur trésor dynastique ${ }^{64}$. Or, son établissement même est ténu, sis sur un rebord escarpé, et n'aurait pu soutenir, selon R.H. Hewsen et E.T. Sinclair, l'existence d'une communauté poliade de quelque importance ${ }^{65}$. Angl aurait donc été une ville-sanctuaire, une nécropole dynastique ainsi qu'une gazophylacie orontide ; possiblement un phylacterion de l'époque achéménide ${ }^{66}$; éventuellement un burg (phrourion) byzantin ${ }^{67}$. Mais elle ne fut jamais une véritable cité ${ }^{68}$.

D'un tout autre caractère, en revanche, était le site d'Amida : par sa position qui commande au plateau septentrional de haute Mésopotamie - le decht de Diyarbakır - par sa conformation urbaine même, il nous paraît davantage correspondre à ce dernier verrou séleucide au Sud de l'Arménie, làmême où une cité grecque d'importance pouvait constituer le point de contrôle le plus efficace sur l'ensemble de la région sub-taurique. Comme on l'a $\mathrm{vu}$, son point topographique le plus élevé (la citadelle d'Içckale) domine sur une très grande distance le territoire avoisinant, à savoir une bonne partie de la Gumathène, la rive gauche du Tigre, et enfin, vers le Nord, les voies descendant du Taurus, au premier chef desquelles l'antique Route Royale, l'axe qu'empruntèrent si souvent les invasions ${ }^{69}$. Une telle fonction de vigilance ne pouvait aucunement être assumée par la localité d'Angl/Egil, encaissée au cœur d'un méandre du Tigre en position aveugle.

\section{CONCLUSION}

Ainsi donc, la confrontation armée eut lieu "au Hanigalbat", autour d'un chef-lieu de la satrapie de Mygdonie-Mésopotamie. Antiochos IV Epiphane devait clairement réaffirmer l'appartenance de toute la haute Mésopotamie - de part et d'autre du Masios - à son royaume, avant de se tourner vers l'Est et le golfe persique pour poursuivre sa restauration territoriale. Dans ce contexte, il nous paraît logique que le roi d'Antioche ait tenu à mettre à profit sa victoire militaire pour installer durablement le site d'Amida comme la dernière cité grecque de Mésopotamie aux confins d'une province définitivement perdue. Trop excentrée, trop isolée topographiquement trop arménienne, surtout - Egil ne pouvait désormais plus jouer un tel rôle. Mais comme on l'a dit, les jeux étaient déjà faits, et cette fondation poliade ne faisait déjà plus figure que de baroud d'honneur pour le dernier roi de la splendeur séleucide ${ }^{70}$. Il s'ensuit qu'Amida ne profita probablement pas très longtemps de ce titre : la mort d'Antiochos, au début de l'hiver 164-163 av. J.-C., referma à jamais l'épisode de la reconquête séleucide en haute Mésopotamie septentrionale.

Désormais, c'était à Rome - et aux Parthes qu'allait avoir affaire Amida de Sophène.

A.P.

64) Toumanoff $1963: 109$ note 68 ; Hewsen $1985: 59$.

65) Ibidem : 59-60: “(...) Angl could never have been a city even by the undemanding standars of antiquity”. Constat partagé par E.T. Sinclair $1989: 358$. gile.

66) C'est l'hypothèse de L. Dillemann (op. cit. : 154) : Egil serait l'ancienne Bara perse. Mais cette identification nous semble fra-

67) Procope (de Aed. II, 4, 22) : Basileon phrourion. Sur quoi, L. Dillemann, op. cit. : 237. Mais l'identification avec Egil, là encore, ne va pas de soi.

68) Selon M. Marciak 2014 : 13, la culture matérielle révèle, à Egil, une sorte de "cas d'école". Il en ira sans doute autrement, à l'époque Arsacide, lorsqu'elle sera promue chef-lieu du district "satrapal" d'Ingilène (Garsoïan 1999 : 10 sq.).

69) C'est d'ailleurs probablement l'une des raisons majeures qui présidèrent au choix que firent, à la fin de l'âge du Bronze, les Araméens du Bit-Zamani d'y établir leur capitale. Sur la Route Royale des Perses et ses vicissitudes dans l'histoire, cf. Assénat et Pérez $2012: 23$ avec la note 71 .

70) Selon Wheeler 2002 : 98, la reconquête éphémère d'Epiphane ne constitua qu'un accident mineur auquel même les Romains n'auraient prêté qu'une attention distraite. 


\section{BIBLIOGRAPHIE}

\section{Sources}

Stephani Byzantii Ethnica, éd. Billerbeck, M. et Zubler, Chr., 2011 : Vol II, Berlin-New-York.

Chronique de Denys de Tell-Mahré : Chabot, J.-B. (éd.), 1895 : quatrième partie. Bibliothèque de l'Ecole des hautes études IV, Sciences historiques et philologiques 112, Paris.

Del Monte, G.F., 1997 : Testi dalla Babilonia ellenistica, Vol. 1. Testi cronografici, Pise-Rome.

Glassner, J.J. (éd. et trad.), 2004 : Chroniques mésopotamiennes, Paris.

Grayson, A.K., 1991 : Assyrian Rulers of the Early First Millenium BC, I (1114-859 BC), (The Royal Inscriptions from Mesopotamia. Assyrian periods v. 2), Toronto.

Isidore de Charax, Mansiones Parthicae = Jacoby, Fragmente, vol. IIIC, Leiden, 1958 : 777-85 ("FgrHist $781 ")$.

Notitia Dignitatum Imperii Romani, Seek, O. (éd.), 1876, Berlin.

Pline l'Ancien, Histoire Naturelle, livre VI, XXX, Panckoucke, Ch.-J. (éd.), 1830, Paris.

Plutarque, Vie de Lucullus, Texte établi et traduit par Flacelière, R. et Chambry, E., 1972, CUF, Paris.

Porphyre apud Jérôme, Commentaire à Daniel (11, 44-45), Patr. Lat. XXV, c. $573=F r$. Gr. Hist., éd. Jacoby, II B 260, frag. 56.

Procope, De Aedificiis, Dewing, H.B. (éd. et trad.), 1940, Loeb C.L., London.

Quinte Curce, Histoires, livre VII-X, Texte établi et traduit par Bardon, H., 1947-1948, CUF, Paris.

Sachs, A.J. et Hunger, H., 1988, 1989 et 1996 : Astronomical Diaries And Related Texts From Babylonia (=AD 1, 2 et 3 ), 3 volumes, Wien.

Strabon, Géographie, XI. Texte établi et traduit par Lasserre, F., 1975, CUF, Paris.

Stückelberger, A. et Grasshoff, G., 2006 : Ptolemaios. Handbuch der Geographie, II, Bâle.

Table de Peutinger, éd. K. Miller, Stuttgart, 1916.

Xénophon, Anabase, Livres I-III, Texte établi et traduit par Masqueray, P., $9^{\mathrm{e}}$ tirage 2009, CUF, Paris.

Zacharie de Mytilène, Chronique, Hamilton, F.J. et Brooks, E.W. (trad.), 1989 : "The syriac chronicle know as that of Zachariah of Mytilene", Londres.

\section{Ouvrages et travaux modernes}

Assénat, M. et Pérez, A., 2012 : “Amida Restituta”, in Gasse, A., Servajean, Fr. et Thiers, Chr. (éds.), Et in Aegypto, et ad Aegyptum, Recueil d'Etudes dédiées à J.C. Grenier, CENiM, Montpellier : 7- 52.

- 2013 : "Epiphaneia... kata Tigre : une fondation séleucide à Amida?", Anatolia Antiqua XXI : 159-166.

Bousdroukis, T., 2003 : "Les noms des colonies séleucides au Proche-Orient”, Topoi, suppl. 4 : 9-24.
Capdetrey, L., 2007 : Le pouvoir séleucide. Territoire, administration, finances d'un royaume hellénistique (312129 av. J.-C.), Paris.

- 2008 : Le domaine séleucide au III siècle, in Grandjean, C. et alii, Le Monde hellénistique, Paris.

Dédéyan, G. (dir.), 2008 : Histoire du Peuple Arménien, Toulouse.

Chaumont, M.-L., 1984 : "Etudes d'Histoire Parthe", Syria 60, $1:$ 63-107.

- 1986-1987 : "'L'Arménie et la route Royale des Perses", REArm, $20:$ 287-307.

- 1993 : "Fondations Séleucides en Arménie Méridionale", Syria $70:$ 431-441.

Clancier, Ph., 2014 : "Antiochos IV dans les sources babyloniennes" in Feyel, Chr. et Graslin-Thomé, L. ( éds.), Le projet politique d'Antiochos IV, Paris : 415438.

Cohen, G.M., 2013 : The Hellenistic Settlements in the East from Armenia and Mesopotamia to Bactria and India, Los Angeles-Londres.

Der Neue Pauly. Historischer Atlas der Antiken Welt, (éd., Olshausen, E., Szydlak, R., Wittke, A.-M.), 2012.

Dillemann, L., 1962 : Haute Mésopotamie orientale et pays adjacents. Contribution à la géographie historique de la région, du $V^{e}$ s. avant l'ère chrétienne au VI ${ }^{e}$. de cette ère, Paris.

Eckardt, K., 1910 : " Die armenischen Feldzüge des Lukullus, II", Klio 10 : 72-115.

Facella, M., 2006 : La dinastia degli Orontidi nella Commagene ellenistico-romana, Pisa.

Fales, Fr.M., 2012 : "'Hanigalbat' in the Early NeoAssyrian Royal Inscriptions: A Retrospective View", in Galil, G. et alii (éds.), The Ancient Near East in the $12^{\text {th }}$ $10^{\text {th }}$ Centuries BCE Culture and History, Proceedings of the International Conference held at the University of Haifa (2-5 May, 2010), Münster : 99-120.

Feissel, D. et Gascou, J., 1989 : "Documents d'archives romains inédits du Moyen Euphrate (III ${ }^{\mathrm{e}}$ s. ap. J.-C.)", CRAI, $133^{\mathrm{e}}$ année, $3: 535-561$.

- 1995 : “Documents d'archives romains inédits du Moyen Euphrate (III ${ }^{\mathrm{e}}$ s. après J.-C.) [I. Les pétitions (T. Euphr. 1 à 5)], Journal des Savants 1-1 : 65-119.

Frankfort, Th., 1963 : "la Sophène et Rome”, Latomus $22: 181-190$.

Freu, J., 2003 : Histoire du Mitanni, Paris

Garelli, P. et Lemaire, A., 1997 : Le Proche-Orient asiatique, T. 2, Les empires mésopotamiens, Israël, Paris, PUF.

Garsöian, N., 1999 : L'Eglise Arménienne et le Grand Schisme d'Orient, Louvain.

Geller, M. et Traina, G., 2013 : "' Tigranu, the Crown Prince of Armenia': Evidence from the Babylonian Astronomical Diaries", KLIO 95 - 2 : 447-454.

Gnoli, T., 1999 : "I papyri dell'Eufrate. Studio di geografia storica", MedAnt II, 1 : 321-358. 
- 2000 : Roma, Edessa e Palmira nel III sec. D.C. Problemi Istituzionali. Uno studio sui Papiri dell'Eufrate, Pisa-Roma.

Hewsen, R.H., 1985 : " $T$ The boundaries of Artaxiad Armenia", REArm $19: 59$.

- 2000 : Armenia. A Historical Atlas, Chicago.

Kupper, J.-R., 1956 : Les nomades en Mésopotamie au temps des rois de Mari, Paris.

Le Rider, G., 1994 : "Antiochos IV (175-164) et le monnayage de bronze séleucide", $B C H$ 118-1 : 17-34.

Lipinski, E., 2000 : The Aramaeans. Their Ancient History, Culture, Religion, Orientalia Lovanensia Analecta 100, Leuven.

Liverani, M., 2008 : La Bible et l'invention de l'histoire. Histoire ancienne d'Israël, Paris.

Marciak, M., 2014 : The Cultural Landscape of Sophene from Hellenistic to Early Byzantine Times, Göttinger Forum für Altertumswissenschaft 17.

Meillet, A. et Benveniste, E., 1931 : Grammaire du vieux perse, Paris.

Mittag, P.F., 2006 : Antiochos IV Epiphanes. Eine politische Biographie, Berlin.

Mutafian, Cl. et Van Lauwe, E., 2001 : Atlas Historique de l'Arménie. Proche-Orient et Sud-Caucase du VIII siècle av. J.-C. au XXI ${ }^{e}$ siècle, Paris.

Petrosyan, A., 2002 : "The Indo-European and Ancient Near Eastern Sources of the Armenian Epic", Journal of Indo-European Studies, Monograph 42, Washington DC.

Radner, K., 2006 : "Provinz. C. Assyrien", Reallexikon der Assyriologie und Vorderasiatischen Archäologie, 11, Berlin - New-York : 42-68.
Radner, K. et Schadner, A., 2001 : "From Tushan to Amedi: Topographical Questions concerning the Upper Tigris Region in the Assyrian Period", in Tuna, N. and Velibeyoğlu, J. (éds.), Salvage Project of the Archaeological Heritage of the Illsu and Carchemish Dam Reservoirs Activities in 1999, Ankara, ODTÜ.

Rostovtzeff, M., 1933 : "Les archives militaires de Doura-Europos", CRAI : 309-323.

Sartre, M., 2001 : D’Alexandre à Zénobie. Histoire du Levant antique (IV siècle avant J.-C. - III siècle après J.-C.), Paris.

Sinclair, E. T., 1989 : Eastern Turkey: An Architectural and Archaeological Survey, Vol. 3, Londres.

Szuchman, J., 2009 : "Bit-Zamani and Assyria", Syria $86: 55-65$.

Talbert, R.J.A. (éd.), 2000 : Barrington Atlas of the Greek and Roman world, Princeton-Oxford.

Toumanoff, C., 1963 : Studies in Christian Caucasian History, Washington.

- 1990 : Les dynasties de la Caucasie chrétienne de l'Antiquité jusqu'au XIX $X^{e}$ siècle : Tables généalogiques et chronologiques, Rome.

Wartke, R.B., 1993 : Urartu - das Reich am Ararat, Kulturgeschichte der antiken Welt 59, Mainz.

Weissbach, F.H., 1927: "Sophene", RE II, 5 :10151019.

Wheeler, E., 2002 : "Southwestern Armenia as a Roman Frontier: Sophene 188 B.C.-299 A.D.", in Hovannisian, R.G. (éd.), Armenian Tsopk/Khapert, Costa Mesa. 


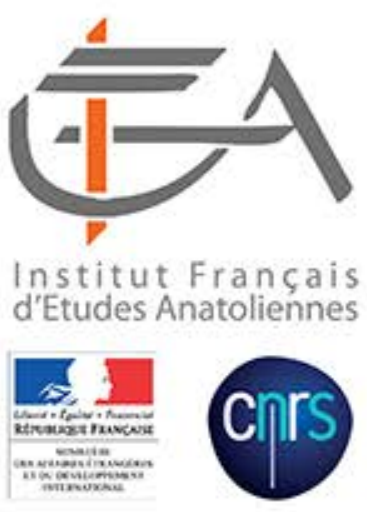

Eurômos : le temple de Zeus vu de l'Est (cliché Sönmez Alemdar).

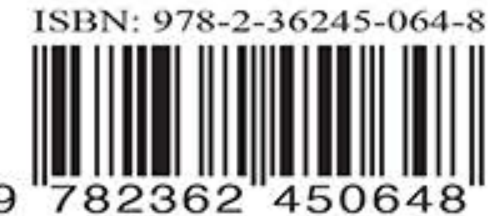

